The Astronomical JouRnaL, 117:2695-2708, 1999 June

(C) 1999. The American Astronomical Society. All rights reserved. Printed in U.S.A.

\title{
STUDY OF THE INTERACTING SYSTEM NGC 6845
}

\author{
IRAPUAN RODRIGUES ${ }^{1,2}$ AND HORACIO DOTTORI ${ }^{2}$ \\ Departamento de Astronomia, Instituto de Física, Universidade Federale do Rio Grande do Sul, CP 15051, Avenue B. Gonçalves 9500, CEP 91501-907 \\ Porto Alegre, Brazil \\ ELIAS BRINKS \\ Universidad de Guanajuato, Apartado Postal 144, Guanajuato, C.P. 36000, Gto, México
}

AND

I. Felix Mirabel

Service d'Astrophysique/CEA, DSM/DAPNIA, Centre d'Etudes de Saclay, 91191 Gif-sur-Yvette, France; and Instituto de Astronomia y Fisica del Espacio, C.C. 67, Suc. 28 (1948), Buenos Aires, Argentina

Received 1998 November 5; accepted 1999 March 5

\begin{abstract}
We present optical spectroscopy, $B, V, R$, and $I$ CCD photometry, and VLA neutral hydrogen observations of the interacting quartet NGC 6845, also know as Klemola 30. NGC $6845 \mathrm{~A}$, the dominant component, sports a broad and bright tidal bridge and a faint tidal tail, which bifurcates. The tidal bridge has a $B-I$ color bluer than that of the inner disk of NGC 6845 A. Five strong condensations, identified as $\mathrm{H}$ II regions brighter than the brightest one known in our Galaxy, are found along the tidal bridge, with the two most luminous located at the bridge tip. Two giant $\mathrm{H}$ II regions, comparable to 30 Dor, are located where the tidal bridge and the tidal tail join the disk of NGC $6845 \mathrm{~A}$. Since the age of the $\mathrm{H}$ II regions is 3-8 Myr, star formation continued to occur along the tidal bridge and in the tidal arm well after they began to be torn apart ( $\geq 100 \mathrm{Myr}$ ). Satoh model fitting to the optical rotation curve of the A component reveals a kinematical mass of $(4.4 \pm 1.2) \times 10^{11} M_{\odot}$, inside the central $12 \mathrm{kpc}$

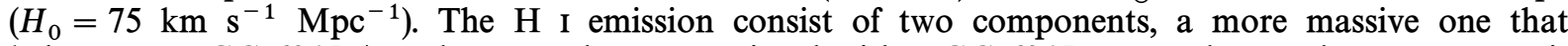
belongs to NGC $6845 \mathrm{~A}$ and a second one associated with NGC $6845 \mathrm{~B}$. We do not detect gas associated with galaxies $\mathrm{C}$ and $\mathrm{D}$. The total amount of $\mathrm{H} \mathrm{I}$ is $1.4 \times 10^{10} M_{\odot}, 5$ time higher than the $\mathrm{H}$ I content of the Milky Way. The $\mathrm{H}$ I kinematics indicates an amount of dark matter associated with the A component 2 times higher than the mass inside its central $12 \mathrm{kpc}$. The group kinematics indicates $M / L \approx 43( \pm 2)(M / L)_{\odot}$ or $M / L \approx 66( \pm 2)(M / L)_{\odot}$, according to two different prescriptions for the internal absorption correction. In spite of this large difference, both values furnish a similar group mass $\left(\approx 1 \times 10^{13} M_{\odot}\right)$. Although preliminary results on $N$-body simulations indicate that either B or C might well create a tidal feature like the bridge of the A component, the collision with B appears to be more favorable.
\end{abstract}

Key words: galaxies: fundamental parameters — galaxies: individual (NGC 6845) -

galaxies: interactions - galaxies: kinematics and dynamics - galaxies: photometry

\section{INTRODUCTION}

Two decades ago, Toomre \& Toomre (1972) demonstrated through numerical models that gravitational interactions between galaxies can generate spectacular tidal features. In earlier work, Zwicky (1956) had already proposed that collisions between giant galaxies may result in the ejection of stars and gas into intergalactic space, resulting in the formation of star clusters and star-forming dwarf galaxies. Direct observational evidence for the existence of such a class of recycled galaxies has already been reported in the literature (see, e.g., Schweizer 1978). Systems like the Antennae (Schweizer 1978; Mirabel, Dottori, \& Lutz 1992) and the Super-antennae (Mirabel, Lutz, \& Maza 1991) show, in addition to stellar material and gas torn from the disks of the interacting galaxies, optically bright clumps along and at the extremes of the tidal tails as well. In some cases there are even chains of knots of bright material

\footnotetext{
${ }^{1}$ Brazilian CNPq fellow.

2 Visiting astronomer, Complejo Astronómico El Leoncito, operated under agreement between the Consejo Nacional de Investigaciones Cientí ficas y Técnicas de la República Argentina and the Universities of La Plata, Córdoba, and San Juan.
}

whose colors become bluer toward the tips (Hibbard et al. 1994). Radio observations of these systems have shown that large amounts of atomic hydrogen are torn away from the parent galaxies, are stretched along the tidal tails, and seem to concentrate at the tips. In contrast, molecular hydrogen is concentrated in the central regions of the parent galaxies. A particularly nice example is the interacting galaxy Arp 105 (Duc \& Mirabel 1994; Duc et al. 1997), which shows near the end of its tidal tail an LMC-like galaxy, resulting from the collision of a spiral with an elliptical galaxy.

It is well known from numerical simulations that the forms and strengths of the tidal bridges and tails depend on the orbital and spin parameters of the colliding galaxies (see, e.g., Toomre \& Toomre 1972; Wright 1972; Eneev, Kozlov, \& Sunyaev 1973; Negroponte \& White 1983; Barnes 1988, 1992). However, the star formation activity within the tidal tails could only recently be numerically studied, after the development of appropriate codes (see, e.g., Hernquist \& Katz 1989; Elmegreen, Kaufmann, \& Thomasson 1993). Models that do not take into account the gas, or the fact that the $\mathrm{H} \mathrm{I}$ is distributed predominantly at the periphery of spirals, are unable to reproduce the observed distribution after a collision (Barnes \& Hernquist 1992), while those that do take these into account find that, 
TABLE 1

Basic Data on the Four Components of the NGC 6845 Quartet (SEe Fig. 1)

\begin{tabular}{cccccl}
\hline \hline Parameter & $\mathrm{A}$ & $\mathrm{B}$ & $\mathrm{C}$ & $\mathrm{D}$ & \multicolumn{1}{c}{ Reference } \\
\hline$\alpha(1950) \ldots \ldots \ldots \ldots$ & 195722.0 & 195729.6 & 195720.3 & 195717.0 & $\mathrm{RC3}$ \\
$\delta(1950) \ldots \ldots \ldots \ldots$ & -471230 & -471153 & -471320 & -471356 & $\mathrm{RC3}$ \\
$l \ldots \ldots \ldots \ldots \ldots \ldots$ & 352.24 & 352.26 & 352.23 & 352.21 & $\mathrm{RC3}$ \\
$b \ldots \ldots \ldots \ldots \ldots \ldots$ & -30.99 & -31.02 & -30.99 & -30.98 & RC3 \\
$m_{B}(\mathrm{mag}) \ldots \ldots \ldots$ & 13.92 & 15.32 & 14.94 & 16.39 & Rose \& Graham 1979 \\
$B_{T}(\mathrm{mag}) \ldots \ldots \ldots \ldots$ & $13.65 \pm 0.15$ & $14.86 \pm 0.15$ & $16.3 \pm 0.30$ & $15.50 \pm 0.20$ & RC3 \\
$m_{B}(\mathrm{mag}) \ldots \ldots \ldots$ & $13.84 \pm 0.02$ & $15.19 \pm 0.02$ & $14.35 \pm 0.02$ & $15.95 \pm 0.02$ & This paper (see Table 4) \\
$V_{\text {sys }}\left(\mathrm{km} \mathrm{s}{ }^{-1}\right) \ldots \ldots$ & 6410 & 6776.5 & 6755 & 7070 & Rose \& Graham 1979 \\
$V_{\text {sys }}\left(\mathrm{km} \mathrm{s}^{-1}\right) \ldots \ldots$ & $6356 \pm 18$ & $6753 \pm 18$ & $7070 \pm 54$ & $6816 \pm 44$ & RC3 \\
$V_{\text {sys }}\left(\mathrm{km} \mathrm{s}^{-1}\right) \ldots \ldots$ & $6399 \pm 20$ & $6746 \pm 20$ & $\ldots$ & $\ldots$ & This paper (see Table 6) \\
\hline
\end{tabular}

Notes. $-V_{\text {sys }}$ is heliocentric. Units of right ascension are hours, minutes, and seconds, and units of declination are degrees, arcminutes, and arcseconds.

after a collision with a massive body, the gas can form huge pools at the extreme ends of the tidal tails (Elmegreen et al. 1993).

In this paper we study the group NGC 6845 (Klemola 30, AM 1957-471). Klemola (1969) described the group as being compact, dominated by a bright, one-armed spiral. In the Arp \& Madore (1987) catalog, NGC 6845 is described as an interacting quartet. Its four components are approximately aligned in a southwest-northeast direction. The components NGC 6845 A and NGC 6845 B, the latter located to the northeast, show abundant signs of ongoing interaction, such as tidal bridges and tails. Many clearly defined knots dot the tidal bridge that links NGC $6845 \mathrm{~A}$ to NGC 6845 B. Signs of interaction seem also to be present in NGC $6845 \mathrm{C}$ and NGC $6845 \mathrm{D}$, according to the sketches of Graham \& Rubin (1973) and Rose \& Graham (1979). In order to study the collision kinematics and the subsequent process of star formation, we carried out optical imaging and spectroscopy as well as radio-synthesis observations.

In $\S 2$ we show our optical and radio observations of NGC 6845 and briefly describe the data reduction; in $\S 3$ we present our analysis; two possible scenarios for the interaction process are discussed in $\S 4$; and in $\S 5$ we summarize our results and present our conclusions.

\section{OBSERVATIONS AND DATA REDUCTION}

Table 1 presents some basic data on NGC 6845. Our observations consist of optical broadband photometry and spectroscopy obtained at the Complejo Astronomico El
Leoncito (CASLEO) ${ }^{3}$ and Bosque Alegre ${ }^{4}$ observatories and $\mathrm{H}$ I observations taken with the Very Large Array. ${ }^{5}$ Tables 2 and 3 list the relevant instrumental and observational parameters. We adopted $H_{0}=75 \mathrm{~km} \mathrm{~s}^{-1} \mathrm{Mpc}^{-1}$ throughout this paper, which results in a distance to NGC 6845 of $85 \mathrm{Mpc}$. At this distance, 1" corresponds to $412 \mathrm{pc}$. The observations are described in more detail below.

TABLE 2

LOG OF BRoADBAND CCD IMAGING OBSERVATIONS

\begin{tabular}{lccc}
\hline \hline Filter & Date & $\begin{array}{c}\text { Number } \\
\text { of Frames }\end{array}$ & $\begin{array}{c}\text { Exposure Time } \\
\text { (s) }\end{array}$ \\
\hline B $\ldots \ldots$. & 1997 Sep 06 & 7 & 2100 \\
V $\ldots \ldots$. & 1997 Sep 06 & 6 & 1800 \\
R ..... & 1997 Sep 06 & 4 & 720 \\
I ..... & 1997 Sep 06 & 4 & 360 \\
\hline
\end{tabular}

${ }^{\mathrm{a}}$ Total integration time.

\footnotetext{
${ }^{3}$ Complejo Astronómico El Leoncito is operated under agreement between the Consejo Nacional de Investigaciones Cientí ficas y Técnicas de la República Argentina and the Universities of La Plata, Córdoba, and San Juan.

${ }^{4}$ Estación Astrofí sica de Bosque Alegre is operated by Observatorio Astronómico of the Universidad Nacional de Córdoba, Argentina.

${ }^{5}$ The VLA is a facility of the National Radio Astronomy Observatory, which is operated by Associated Universities, Inc., under cooperative agreement with the National Science Foundation.
}

TABLE 3

LOG OF SPECTROSCOPIC OBSERVATIONS

\begin{tabular}{|c|c|c|c|c|c|c|c|c|}
\hline Position & Date & Spectrograph & $\begin{array}{c}\text { Grating } \\
\text { (lines } \mathrm{mm}^{-1} \text { ) }\end{array}$ & $\begin{array}{c}\text { Spectral Range } \\
(\AA ̊)\end{array}$ & $\begin{array}{c}\text { Resolution } \\
(\AA)\end{array}$ & $\begin{array}{l}\text { Slit Size } \\
\text { (arcsec) }\end{array}$ & $\begin{array}{l}\text { Number of } \\
\text { Frames }\end{array}$ & $\begin{array}{l}\text { Total Exposure } \\
\text { Time } \\
\text { (s) }\end{array}$ \\
\hline $1 \ldots \ldots \ldots$ & 1994 Jul 08 & $B \& C$ & 300 & $4100-6900$ & 8 & $4.5 \times 170$ & 3 & 4800 \\
\hline $2 \ldots \ldots \ldots$ & 1994 Jul 08 & $B \& C$ & 300 & $4100-6900$ & 8 & $6 \times 170$ & 3 & 10800 \\
\hline $2 \ldots \ldots \ldots$ & 1997 Sep 08 & REOSC & 600 & $5870-7460$ & 5 & $3.6 \times 120$ & 1 & 3600 \\
\hline $2 * \ldots \ldots$ & 1997 Dec 18 & Multifunction & 1200 & $6130-6850$ & 2 & $1 \times 130$ & 1 & 3600 \\
\hline $3 \ldots \ldots$ & 1994 Jul 09 & $B \& C$ & 300 & $4100-6900$ & 10 & $2 \times 170$ & 3 & 3200 \\
\hline $4 \ldots \ldots \ldots$ & 1994 Jul 10 & REOSC & 300 & $3700-7150$ & 8 & $2 \times 120$ & 2 & 1800 \\
\hline $5 \ldots \ldots \ldots$ & 1994 Jul 10 & REOSC & 300 & $3700-7150$ & 8 & $6 \times 120$ & 2 & 2700 \\
\hline $6 \ldots \ldots$ & 1997 Sep 08 & REOSC & 600 & $5870-7460$ & 6 & $2.3 \times 120$ & 4 & 9300 \\
\hline $7 \ldots \ldots$ & 1997 Sep 08 & REOSC & 600 & $5870-7460$ & 6 & $2.3 \times 120$ & 1 & 3600 \\
\hline
\end{tabular}

NoTES.-Slit positions given in the first column are those marked in Fig. 5. The spectrum marked with an asterisk was observed at the Bosque Alegre Astrophysical Station; all other observations were performed at CASLEO. 


\subsection{Optical Images}

Broadband $B, V, R$, and $I$ CCD images were obtained in 1997 September with the Ritchey-Chrétien $2.15 \mathrm{~m}$ telescope at CASLEO, Argentina, using a thinned Tektronix $1024 \times 1024$ pixel CCD detector. The telescope focal ratio is $\mathrm{f} / 8$. The final image scale, with a focal reducer, is 0 ". 81 $\operatorname{pixel}^{-1}$. The seeing during the observations varied between 2 " and 4".5. Data reduction was performed with IRAF, using standard procedures. Different frames taken through the same filter were registered to a common center, using field stars as a reference, and co-added to remove cosmic ray events and increase the dynamic range. Small seeing differences among frames were corrected by Gaussian filtering. Flux calibration was based on standard stars from Landolt (1992). We corrected as usual for atmospheric extinction.

\subsection{Optical Spectroscopy}

Long-slit spectroscopic observations were carried out in 1994 July (seeing $\left.=11^{\prime \prime} .7\right)$ and 1997 September $\left(\right.$ seeing $\left.=2^{\prime \prime}\right)$ with the $2.15 \mathrm{~m}$ telescope of the CASLEO observatory and in 1997 December with the Bosque Alegre $1.52 \mathrm{~m}$ telescope (seeing $=1$ 1.5). In Table 3 we present the log of the spectroscopic observations. The slit positions, given in the first column of this table, are marked in Figure 5.

Data processing was again done with IRAF following standard methods for bias subtraction, flat fielding, and image combination. The resulting two-dimensional spectra were wavelength calibrated using $\mathrm{HeNeAr}$ comparison spectra and flux calibrated using spectrophotometric standard stars from Stone \& Baldwin (1983). Galactic extinction was corrected with the standard absorption law, adopting $A_{B}=0.16$ (Burstein \& Heiles 1984).

\subsection{VLA Radio Observations}

VLA D-array observations of the NGC 6845 system were obtained during a short $1.5 \mathrm{hr}$ run on 1996 June 19. Because of its far southern declination, the synthesized beam is substantially elongated in the north-south direction. The beam measures $112^{\prime \prime} \times 37^{\prime \prime}$, at a +14.5 angle measured from north to east. The sensitivity of the system was rather modest, and the $\mathrm{H}$ I data should be seen as exploratory. As it was not obvious at what velocities $\mathrm{H}$ I could be expected, we aimed for velocity coverage rather than for velocity resolution, trying to maximize our sensitivity for the velocity range expected for objects A and B (radial velocities are given in Table 1). The velocity resolution was $43.1 \mathrm{~km} \mathrm{~s}^{-1}$, covering the interval $6450 \mathrm{~km} \mathrm{~s}^{-1}$ to $6900 \mathrm{~km} \mathrm{~s}^{-1}$ with both right-hand (R) and left-hand (L) polarization; the intervals from $6000 \mathrm{~km} \mathrm{~s}^{-1}$ to $6450 \mathrm{~km} \mathrm{~s}^{-1}$ and $6900 \mathrm{~km} \mathrm{~s}^{-1}$ to 7050 $\mathrm{km} \mathrm{s}^{-1}$ were observed with one polarization only. Data calibration and mapping was performed with AIPS. The final maps have an rms noise of $1.6 \mathrm{mJy}^{\text {beam }}{ }^{-1}$ or $0.23 \mathrm{~K}$ (where $1 \mathrm{mJy}^{\text {beam }}{ }^{-1}$ corresponds to $0.15 \mathrm{~K}$ ).

\section{RESULTS}

\subsection{Optical Morphology and Photometry}

We present in Figure 1 the $\mathrm{R}$ image, enhancing the lowest surface brightness levels in order to highlight the faint details that might have been produced by the interaction. Clear indications of strong collision are observed in NGC $6845 \mathrm{~A}$ and NGC $6845 \mathrm{~B}$. The tidal bridge between galaxies A and B presents a fainter counterpart, a tidal tail that bifurcates and seems to connect to components C and D (see sketches in Graham \& Rubin 1973 and Rose \& Graham 1979; Plate I in Rose 1979).

In Table 4 we present the integrated $B$ magnitude and colors $B-V, V-R$, and $V-I$ of the group members. As can be seen from Table 1, our $B$ magnitudes are in better agreement with Rose \& Graham (1979) than with RC3. The $B-I$ color map (Fig. 2) shows that the color of the tidal bridge is bluer than the inner disk of the A and B components and comparable to their outer disks. Hibbard et al. (1994) pointed out that in the case of the evolved merge NGC 7252 the tidal tails are $0.2-0.5$ mag bluer in $B-R$ than the outer regions of the main body.

NGC $6845 \mathrm{~A}$ is classified as $\mathrm{SB}(\mathrm{s}) \mathrm{b}$ (RC3). The color distribution along its bar is not uniform. To the southwest, the bar is much redder, indicating that on this side we are seeing dust in front of the stellar bar, where it is usually accumulated in more or less linear shock fronts. The bar of NGC 6845 $\mathrm{A}$ is shifted by $30^{\circ}$ counterclockwise with respect to the photometric major axis, and it may have been tidally created by the encounter, as suggested for example by Barnes (1988) or the Salo (1991) simulations of disk-disk encounters.

Plumes and small condensations are evident around NGC $6845 \mathrm{~B}$ and are likely debris of the tidal interaction. The tidal bridge linking the NGC $6845 \mathrm{~A}$ and $\mathrm{B}$ galaxies is very pronounced and knotty (Fig. 3). The knots are H II regions, as will be shown in $\S 3.2$.

The Rose (1979) CTIO $4 \mathrm{~m}$ plate shows more clearly than our Figure 1 the presence of condensations in the tidal tail, especially a set of them close to the northwest side of NGC $6845 \mathrm{C}$, although it does not shows the bridge knotty struc-

TABLE 4

INTEGRATED $B$ MAGNitudes AND COLORS OF THE GROUP MEMBERS

\begin{tabular}{|c|c|c|c|c|c|c|c|c|c|c|c|c|}
\hline $\begin{array}{l}\text { Galaxy } \\
\text { (1) }\end{array}$ & $\begin{array}{l}B \\
(2)\end{array}$ & $\begin{array}{c}(B-V) \\
\text { (3) }\end{array}$ & $\begin{array}{c}(V-R) \\
\quad(4)\end{array}$ & $\begin{array}{c}(V-I) \\
(5)\end{array}$ & $\begin{array}{l}b / a \\
(6)\end{array}$ & $\begin{array}{c}\Delta m_{B} \\
\text { (7) }\end{array}$ & $\begin{array}{c}\Delta m_{i}^{(1)} \\
(8)\end{array}$ & $\begin{array}{c}\Delta m_{i}^{(2)} \\
(9)\end{array}$ & $\begin{array}{c}M_{B}^{(1)} \\
(10)\end{array}$ & $\begin{array}{c}M_{B}^{(2)} \\
(11)\end{array}$ & $\begin{array}{l}L_{B}^{(1)} \\
(12)\end{array}$ & $\begin{array}{l}L_{B}^{(2)} \\
(13)\end{array}$ \\
\hline A & $13.84 \pm 0.02$ & $0.64 \pm 0.03$ & $0.23 \pm 0.07$ & $0.20 \pm 0.14$ & $0.54 \pm 0.06$ & 0.16 & 0.50 & 0.32 & -21.56 & -21.38 & $3.2 \times 10^{40}$ & $2.7 \times 10^{40}$ \\
\hline B & $15.19 \pm 0.02$ & $0.64 \pm 0.03$ & $0.26 \pm 0.10$ & $0.29 \pm 0.22$ & $1 \pm 0.08$ & 0.16 & 0.00 & 0.10 & -19.71 & -19.81 & $5.9 \times 10^{39}$ & $6.5 \times 10^{39}$ \\
\hline $\mathrm{C} \ldots \ldots$ & $14.35 \pm 0.02$ & $0.81 \pm 0.04$ & $0.35 \pm 0.10$ & $0.16 \pm 0.17$ & $0.22 \pm 0.05$ & 0.16 & 1.86 & 0.82 & -22.41 & -21.37 & $7.1 \times 10^{40}$ & $2.7 \times 10^{40}$ \\
\hline $\mathrm{D} \ldots \ldots$ & $15.95 \pm 0.02$ & $0.83 \pm 0.04$ & $0.35 \pm 0.08$ & $0.11 \pm 0.18$ & $0.33 \pm 0.03$ & 0.16 & 1.06 & 0.50 & -20.01 & -19.45 & $7.8 \times 10^{39}$ & $4.6 \times 10^{39}$ \\
\hline
\end{tabular}

Notes.-Integrated B magnitudes and colors in Johnson's system. All galaxies were measured through a 64"8 aperture. Col. (1): NGC 6845 group individual galaxy names. Col. (2): Apparent blue magnitudes. Cols. (3), (4), and (5): Colors. Col. (6): Corrected inclination angle (see $\S 3.4 .4)$. Col. (7): Corrections to apparent B magnitude for Galactic extinction (Burstein \& Heiles 1984). Cols. (8) and (9): Internal extinction corrections to face-on magnitudes, following Rubin et al. 1982 and RC3, respectively (see § 3.4.4). Cols. (10) and (11): Absolute blue magnitudes with corrections for internal absorption given by $\Delta m_{i}^{(1)}$ and $\Delta m_{i}^{(2)}$, respectively. We used the mean Galactocentric velocity of the group members as the distance indicator. Cols. (12) and (13): Absolute blue luminosity (ergs s${ }^{-1}$ ) as taken from cols. (10) and (11), respectively $\left(H_{0}=75 \mathrm{~km} \mathrm{~s}^{-1} \mathrm{Mpc}^{-1}\right)$. 


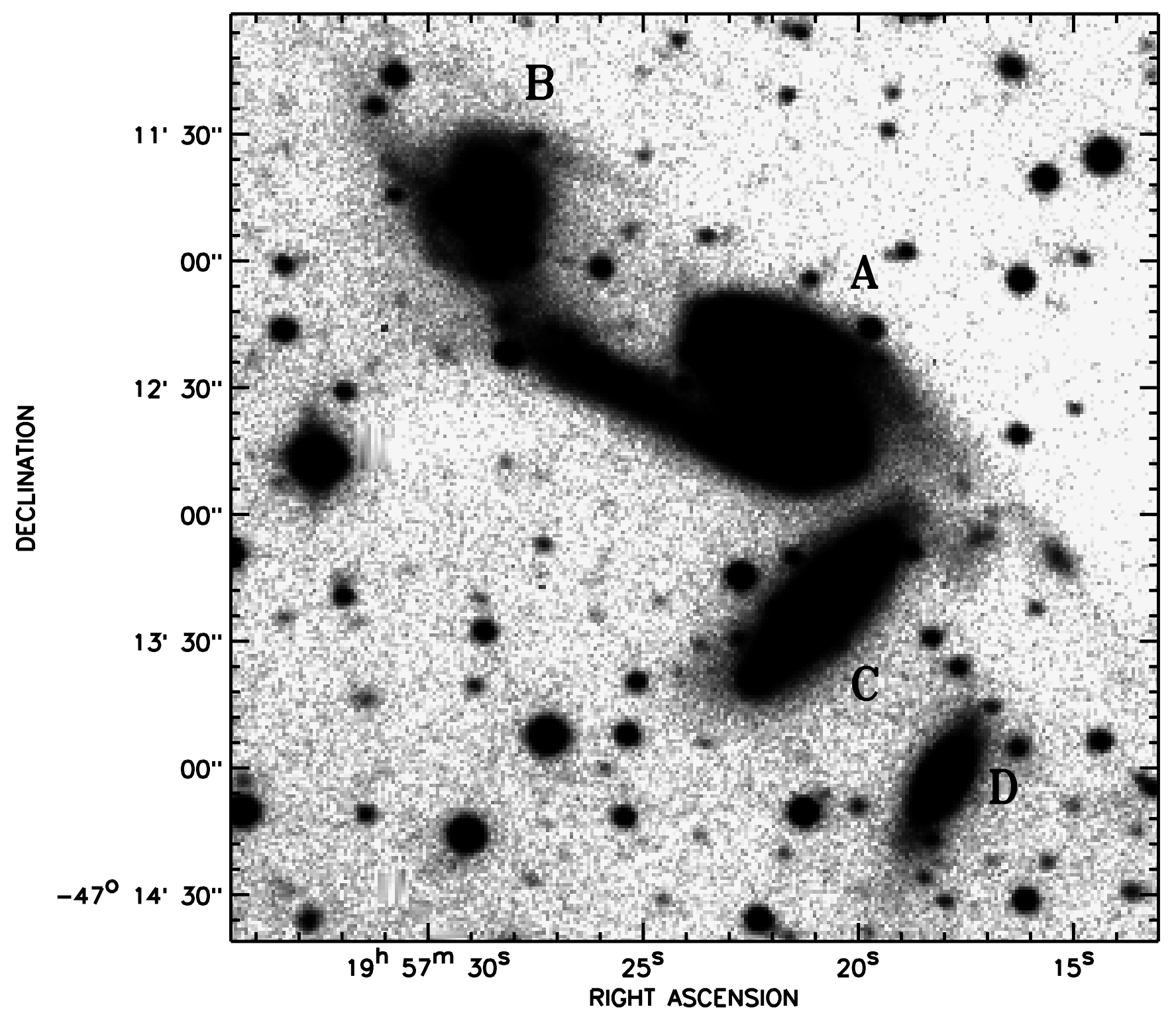

FIG. 1. $-600 \mathrm{~s} \mathrm{R}$-band image of the group of galaxies NGC 6845 or Klemola 30 . The contrast is chosen so as to emphasize low surface-brightness levels. A very good photo of this group can be seen in Rose (1979).

ture because it is overexposed. Based on Rose's plate, Rose \& Graham (1979) schematically suggested that the NGC 6845 A western tidal tail bifurcates and links to the galaxies NGC 6845 C and D.

\subsection{Knots, Their Brightness and Colors}

Table 5 shows the $V$ magnitude and $B-V, V-R$, and $V-I$ color indexes for the most conspicuous knots marked in Figure 3. Without exception the knots present an H IIregion like spectrum, as shown in Figure 4, and we feel therefore confident in identifying them as regions of recent star formation. In order to correct the colors for internal absorption, we used for all regions the mean value of the ratio of $\mathrm{H} \alpha$ to $\mathrm{H} \beta$ as was measured in knots 4 , 7, and 8 (see Table 6). $\mathrm{H} \alpha$ and $\mathrm{H} \beta$ intensities were measured from slit positions 3 and 5 for knot 4 and slit position 4 for knots 7 and 8 (see Fig. 5). Although these regions are located at very different positions within the system, they present roughly the same $\mathrm{H} \alpha / \mathrm{H} \beta$, so we assume a constant $\mathrm{H} \alpha / \mathrm{H} \beta$ of 4.3 to correct the other regions through the formula

$$
A_{\lambda}=\left(\frac{4.69}{\lambda}-1.45\right) \log \left(\frac{\mathrm{H} \alpha / \mathrm{H} \beta}{2.88}\right)
$$

(Osmer, Smith, \& Weedman 1974).

We compared the brightness and colors of the knots to the evolutionary synthesis models of Bertelli et al. (1994). We chose from their models those with a Salpeter initial mass function (IMF) and a heavy element abundance $Z=$ $Z_{\odot}$. In Table 5 we present the mean age of the knots obtained by fitting our three colors to Bertelli et al. models. Their masses were derived from the $V$ magnitudes and assuming a distance of $85 \mathrm{Mpc}$. Within the errors all the knots, except for numbers 6 and 8, are coeval to within our stated accuracy. The derived values, quoted in Table 5, imply that we are dealing with star clusters with masses $\leq 5 \times 10^{5} M_{\odot}$.

In Table 6 we present the $\mathrm{H} \alpha$ emission-line luminosities of all the knots in Figure 3, plus the nuclear emission of 


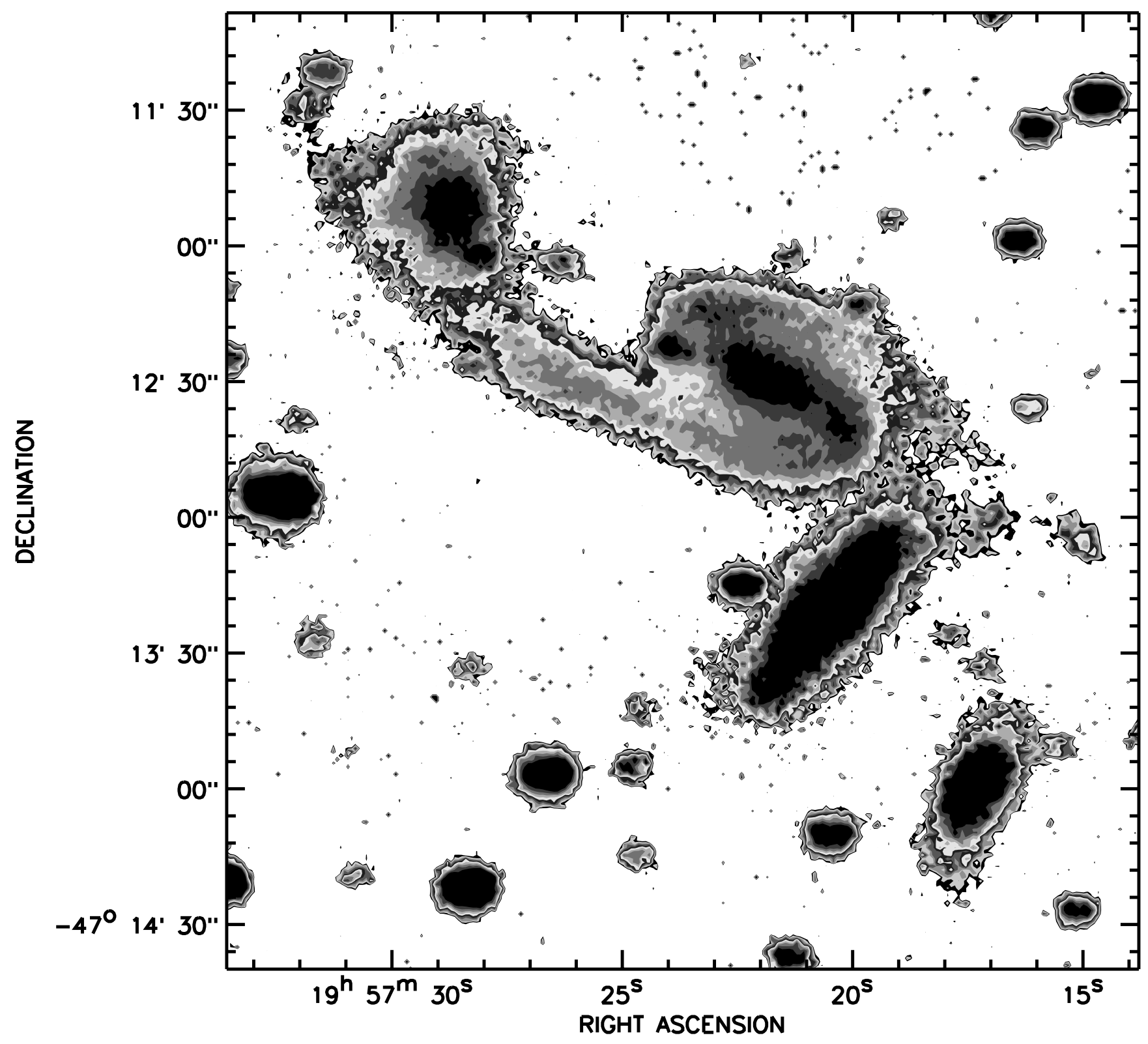

FIG. 2. $-B-I$ color map. 10 gray levels are shown, ranging from 0.5 (white) to $3.5 \mathrm{mag} \mathrm{pixel}^{-1}$ (black)

TABLE 5

Integrated $V$ Magnitudes and Colors for the Condensations DesCribed in the TeXt (SEE ALSO FIG. 3)

\begin{tabular}{|c|c|c|c|c|c|c|}
\hline Region & $V$ & $(B-V)$ & $(V-R)$ & $(V-I)$ & $\begin{array}{c}\text { Age } \\
\left(10^{6} \mathrm{yr}\right)\end{array}$ & $\begin{array}{c}\text { Mass } \\
\left(10^{4} M_{\odot}\right)\end{array}$ \\
\hline 1 & $16.38 \pm 0.02$ & $0.17 \pm 0.04$ & $0.06 \pm 0.09$ & $0.27 \pm 0.11$ & $6.9 \pm 1.0$ & $21 \pm 5$ \\
\hline $2 \ldots \ldots$ & $16.43 \pm 0.04$ & $0.15 \pm 0.08$ & $-0.02 \pm 0.12$ & $0.12 \pm 0.22$ & $6.3 \pm 1.0$ & $20 \pm 5$ \\
\hline $3 \ldots \ldots$ & $16.45 \pm 0.01$ & $0.20 \pm 0.02$ & $0.00 \pm 0.12$ & $0.15 \pm 0.13$ & $6.5 \pm 1.0$ & $19 \pm 5$ \\
\hline $4 \ldots \ldots$ & $16.03 \pm 0.02$ & $0.28 \pm 0.03$ & $-0.05 \pm 0.08$ & $-0.30 \pm 0.16$ & $6.6 \pm 1.0$ & $28 \pm 5$ \\
\hline $5 \ldots \ldots$ & $16.18 \pm 0.02$ & $0.26 \pm 0.03$ & $0.05 \pm 0.06$ & $-0.28 \pm 0.12$ & $6.6 \pm 1.0$ & $25 \pm 5$ \\
\hline $6 \ldots \ldots$ & $18.52 \pm 0.06$ & $0.27 \pm 0.09$ & $0.11 \pm 0.19$ & $0.50 \pm 0.43$ & $8.0 \pm 1.0$ & $1.3 \pm 5$ \\
\hline $7 \ldots \ldots$ & $14.57 \pm 0.01$ & $0.26 \pm 0.02$ & $-0.13 \pm 0.05$ & $-0.48 \pm 0.09$ & $5.3 \pm 1.0$ & $51 \pm 5$ \\
\hline $8 \ldots \ldots$ & $14.81 \pm 0.01$ & $-0.37 \pm 0.02$ & $-0.10 \pm 0.06$ & $-0.40 \pm 0.09$ & $<3.0 \pm 1.0$ & $40 \pm 5$ \\
\hline
\end{tabular}

NoTES.-All regions were measured through a 4" aperture. Magnitudes were corrected for Galactic extinction with the standard absorption law, adopting $A_{B}=0.16$ (Burstein \& Heiles 1984) and for internal absorption using $\mathrm{H} \alpha / \mathrm{H} \beta=4.3$ (see $\S 3.2$ ). 

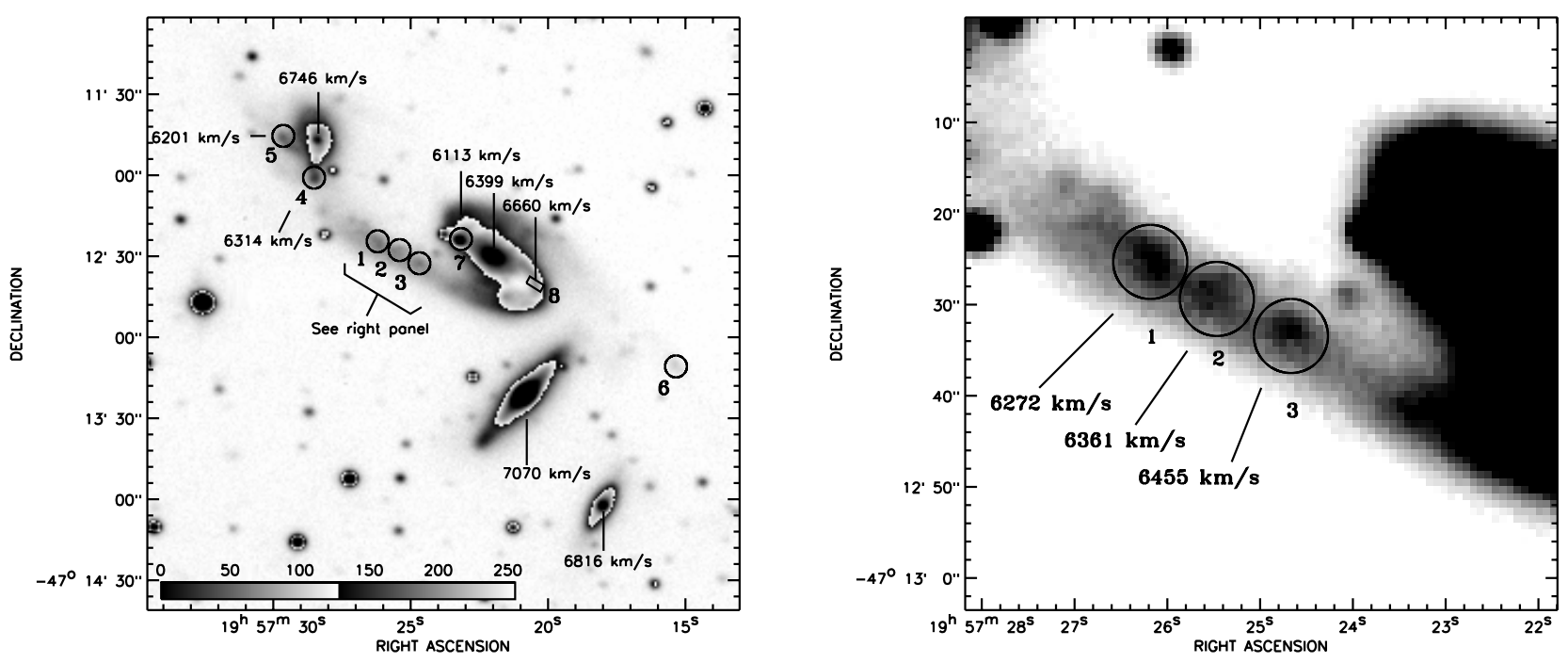

FIG. 3.-Same as Fig. 1, detaching bright condensations. The circles show the positions of the H II regions whose photometric and spectroscopic measurements are presented in Tables 5 and 6. Left panel: Radial velocities of the galaxies A, B, C, and D, as well as of some selected positions. Right panel: Detail of the tidal bridge, showing $\mathrm{H}$ in regions 1,2 , and 3.
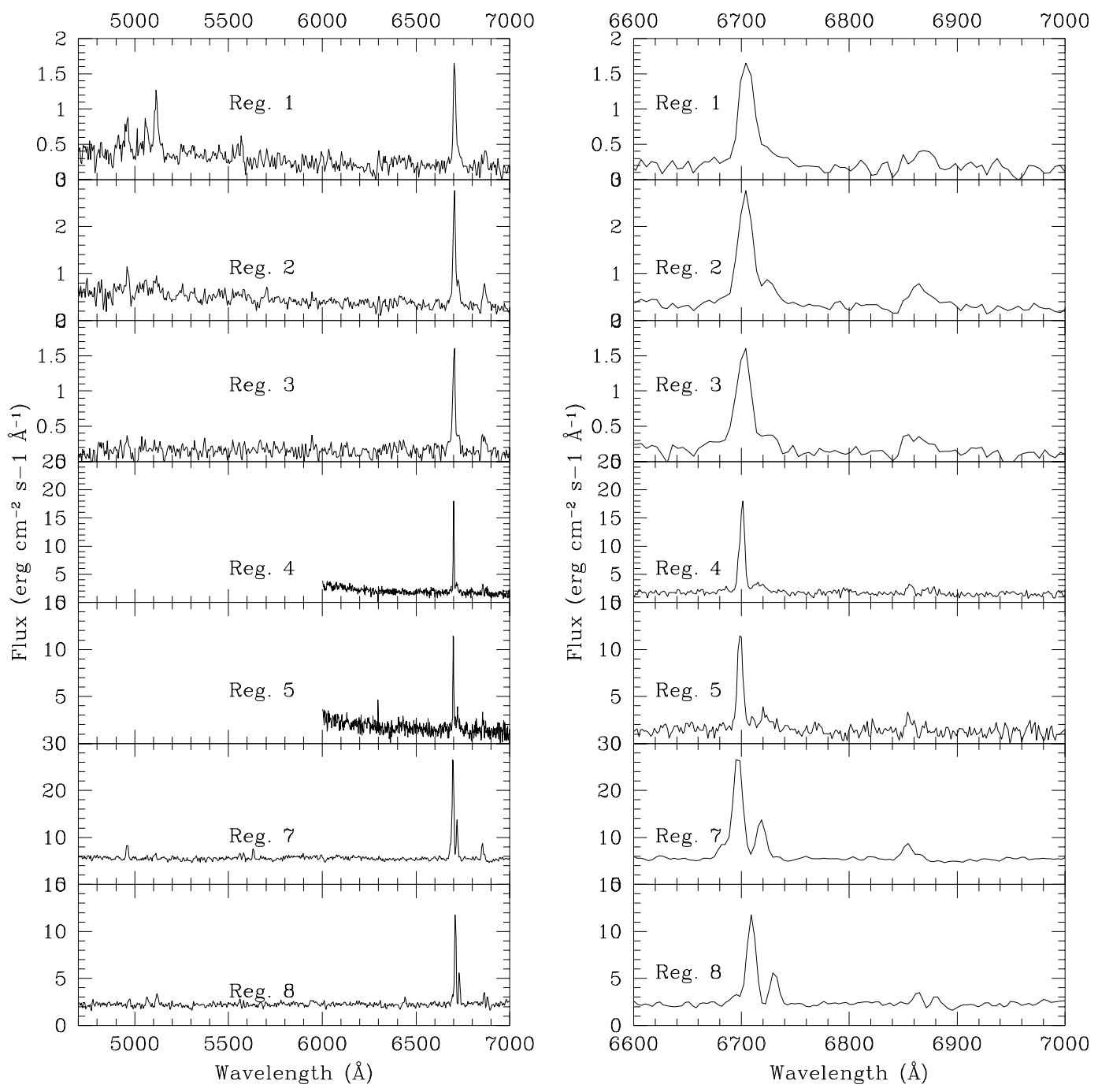

FIG. 4. - Composite image showing our optical spectra toward seven of the eight $\mathrm{H}$ in regions indicated in Table 5 and Fig. 3. Left panel: Entire observed wavelength range. Right panel: Spectral region around $\mathrm{H} \alpha$ and $[\mathrm{S}$ II] $\lambda \lambda 6717,6731$. 
TABLE 6

H $\alpha$ Luminosities, $\mathrm{H} \alpha$-to-H $\beta$ Ratios, Slit Dimensions and Positions, and Radial VELOCITIES FOR THE CONDENSATIONS IN FIGURE 3

\begin{tabular}{|c|c|c|c|c|c|}
\hline $\begin{array}{l}\text { Region } \\
\text { (1) }\end{array}$ & $\begin{array}{c}L_{\mathbf{H} \alpha} \\
\left(\operatorname{ergs~s^{-1}}\right) \\
(2)\end{array}$ & $\begin{array}{c}\mathrm{H} \alpha / \mathrm{H} \beta \\
\text { (3) }\end{array}$ & $\begin{array}{l}\text { Slit Dimension } \\
\text { (arcsec) } \\
(4)\end{array}$ & $\begin{array}{c}\text { Slit } \\
\text { Position(5) }\end{array}$ & $\begin{array}{c}\text { Velocity } \\
\left(\mathrm{km} \mathrm{s}^{-1}\right) \\
(6)\end{array}$ \\
\hline $1 \ldots \ldots \ldots \ldots \ldots \ldots$ & $2.3 \times 10^{39}$ & ... & $6(1) \times 6$ & 2 & 6272 \\
\hline $2 \ldots \ldots \ldots \ldots \ldots \ldots \ldots$ & $3.0 \times 10^{39}$ & $\ldots$ & $6(1) \times 6$ & 2 & 6361 \\
\hline $3 \ldots \ldots \ldots \ldots \ldots \ldots$ & $1.8 \times 10^{39}$ & $\ldots$ & $6(1) \times 6$ & 2 & 6455 \\
\hline $4 \ldots \ldots \ldots \ldots \ldots \ldots \ldots$ & $9.1 \times 10^{39}$ & 4.2 & $2.3 \times 6$ & $7,3,5$ & 6314 \\
\hline $5 \ldots \ldots \ldots \ldots \ldots \ldots$ & $6.5 \times 10^{39}$ & $\ldots$ & $2.3 \times 6$ & 6 & 6201 \\
\hline $6 \ldots \ldots \ldots \ldots \ldots \ldots$ & $\ldots$ & $\ldots$ & $\ldots$ & $\ldots$ & $\ldots$ \\
\hline $7 \ldots \ldots \ldots \ldots \ldots \ldots$ & $2.2 \times 10^{40}$ & 4.1 & $2 \times 6.2$ & 4 & 6113 \\
\hline $8 \ldots \ldots \ldots \ldots \ldots \ldots \ldots$ & $8.7 \times 10^{39}$ & 4.6 & $2 \times 6$ & 4 & 6660 \\
\hline NGC 6845 A...... & $7.7 \times 10^{39}$ & $\ldots$ & $2 \times 10$ & 4 & 6399 \\
\hline NGC 6845 B ...... & $1.1 \times 10^{40}$ & $\ldots$ & $2.3 \times 8$ & 6,7 & 6746 \\
\hline
\end{tabular}

Notes.-Col. (1): Region label as in Table 5 and Fig. 3. Cols. (2) and (3): $\mathrm{H} \alpha$ luminosities and $\mathrm{H} \alpha$-to-H $\beta$ ratios, respectively. Col. (4): Slit dimensions. The numbers in parentheses on the slit dimension of regions 1,2 , and 3 correspond to the slit width of the spectrum used to measure velocities, while the values outside the parentheses are for the spectra used to measure flux. Col. (5): Slit position, as in Table 3. In region 4, three slit positions were used: position 7 to measure $\mathrm{H} \alpha$ flux and radial velocity, and positions 3 and 5 to measure the $\mathrm{H} \alpha$-to- $\mathrm{H} \beta$ ratio. Col. (6): Heliocentric radial velocity of the region.

NGC 6845 A and NGC 6845 B. As can be seen, knots 1, 2, and 3 , located in the middle of the tidal bridge, have $\mathrm{H} \alpha$ luminosities comparable to that of NGC 3603, the most luminous $\mathrm{H}$ II region known to exist in our Galaxy $\left(L_{\mathbf{H} \alpha}=\right.$ $1.1 \times 10^{39} \mathrm{ergs} \mathrm{s}^{-1}$; Melnick, Tapia, \& Terlevich 1989) or higher. Regions 4 and 5, at the tip of the tidal bridge are even brighter, being similar to 30 Doradus $\left(L_{\mathrm{H} \alpha}=6.3\right.$ $\times 10^{39}$ ergs s$^{-1}$; Kennicutt, Edgar, \& Hodge 1989). Giant $\mathrm{H}$ II regions near the ends of tidal bridges and tails were reported for various systems, as in NGC 4676 by Stockton (1974) and in NGC 2623, NGC 6621/22, NGC 3256 and NGC $2535 / 36$ by Schweizer (1978). These ionizing clusters are usually more luminous than the ones along the tidal tails. Finally, $\mathrm{H}$ II regions 7 and 8 , located at the extreme ends of the bar in NGC $6845 \mathrm{~A}$, have luminosities comparable to the "jumbo" $\mathrm{H}$ II region NGC $3310\left(L_{\mathbf{H} \alpha}=1.4\right.$ $\times 10^{40}$ ergs s$^{-1}$, Pastoriza et al. 1993), indicating strong star formation activity in these places, which may also have been triggered by the collision (Kennicutt \& Chu 1988).

Like other interacting systems, this group is prominent in the infrared. The IRAS fluxes $\left(F_{12 \mu \mathrm{m}}=0.35 \mathrm{Jy}, F_{25 \mu \mathrm{m}}=\right.$ $0.32 \mathrm{Jy}, F_{60 \mu \mathrm{m}}=2.78 \mathrm{Jy}$, and $F_{100 \mu \mathrm{m}}=6.66 \mathrm{Jy} ;$ Rush, Malkan \& Spinoglio 1993) lead to a group total luminosity of $L_{\mathrm{IR}}=7.9 \times 10^{10} L_{\odot}$, locating this group at the fainter end of luminous IR galaxies according to the definition by Sanders \& Mirabel (1996).

\subsection{H I Gas Distribution}

In Figure 6 we show the $\mathrm{H}$ I isodensity contours superimposed on the $R$-band image. Despite the low spatial resolution of the radio observations, it is possible to distinguish two velocity components that differ by a few hundred kilometers per second, a main component centered on NGC 6845 A and a secondary one associated with NGC $6845 \mathrm{~B}$. No H I was detected at the velocities of galaxies C and $\mathrm{D}$.

The main H I component presents a clear extension toward the southwest, bending south, following the stellar tidal tail. To the east, it shows a protrusion that coincides with the bright optical tidal bridge, with velocities compat- ible with this feature within the observational errors. The integrated $\mathrm{H}$ I flux of the main component is $6.79 \mathrm{Jy} \mathrm{km} \mathrm{s}{ }^{-1}$, which translates to an $\mathrm{H}$ I mass of $1.15 \times 10^{10} M_{\odot}$.

The component associated with NGC $6845 \mathrm{~B}$ is virtually stationary in velocity. The mean velocity is around $6700 \mathrm{~km}$ $\mathrm{s}^{-1}$, close to that of NGC $6845 \mathrm{~B}$. Spatially, it is slightly shifted eastward with respect to NGC $6845 \mathrm{~B}$. The integrated $\mathrm{H}$ I flux is $1.52 \mathrm{Jy} \mathrm{km} \mathrm{s}{ }^{-1}$, which translates to $2.58 \times 10^{9} M_{\odot}$.

The total integrated $\mathrm{H}$ I flux of both components is 8.31 Jy km s${ }^{-1}$, corresponding to an $\mathrm{H}$ I mass of $1.41 \times 10^{10}$ $M_{\odot}$, or about 5 times more $\mathrm{H}$ I than is contained in the Milky Way.

\subsection{Kinematics}

In Figure 4 we show the slit positions along which we obtained spectra. For ease of reference, we indicated in Figure 3 the radial velocity of the more relevant objects. Radial velocities were obtained using a weighted mean of the values measured for the $\mathrm{H} \alpha, \mathrm{H} \beta$, and [O III] $5007 \AA$ lines. A comparison with Table 1 shows that the radial velocity values we measured for NGC $6845 \mathrm{~A}$ and B are in agreement with previous results from Rose \& Graham (1979) and RC3. There is, however, a discrepancy between the values given by the cited references for the radial velocities of components C and D. Apparently one of these sources made a mistake in the galaxies' identification.

\subsubsection{NGC 6845 A: Optical Rotation Curve}

The rotation curve of NGC $6845 \mathrm{~A}$, presented in Figure 7, was obtained from slit position 4 (see Table 3 and Fig. 4), along the apparent major axis of the galaxy. Line-of-sight velocity measurements were transformed to circular velocities adopting an inclination angle of $57^{\circ}\left( \pm 3^{\circ}\right)$, derived by fitting ellipses to the optical isophotes of the $B$ image (using the appropriated procedure in the STSDAS-IRAF package). Since the isophotes in the inner part of NGC 6845 A are influenced by the bar profile, and those more external are tidally distorted, we adopted the ellipticity of isophotes at intermediate radii as the best indicator for the inclination. There is a marked difference between the northeast 


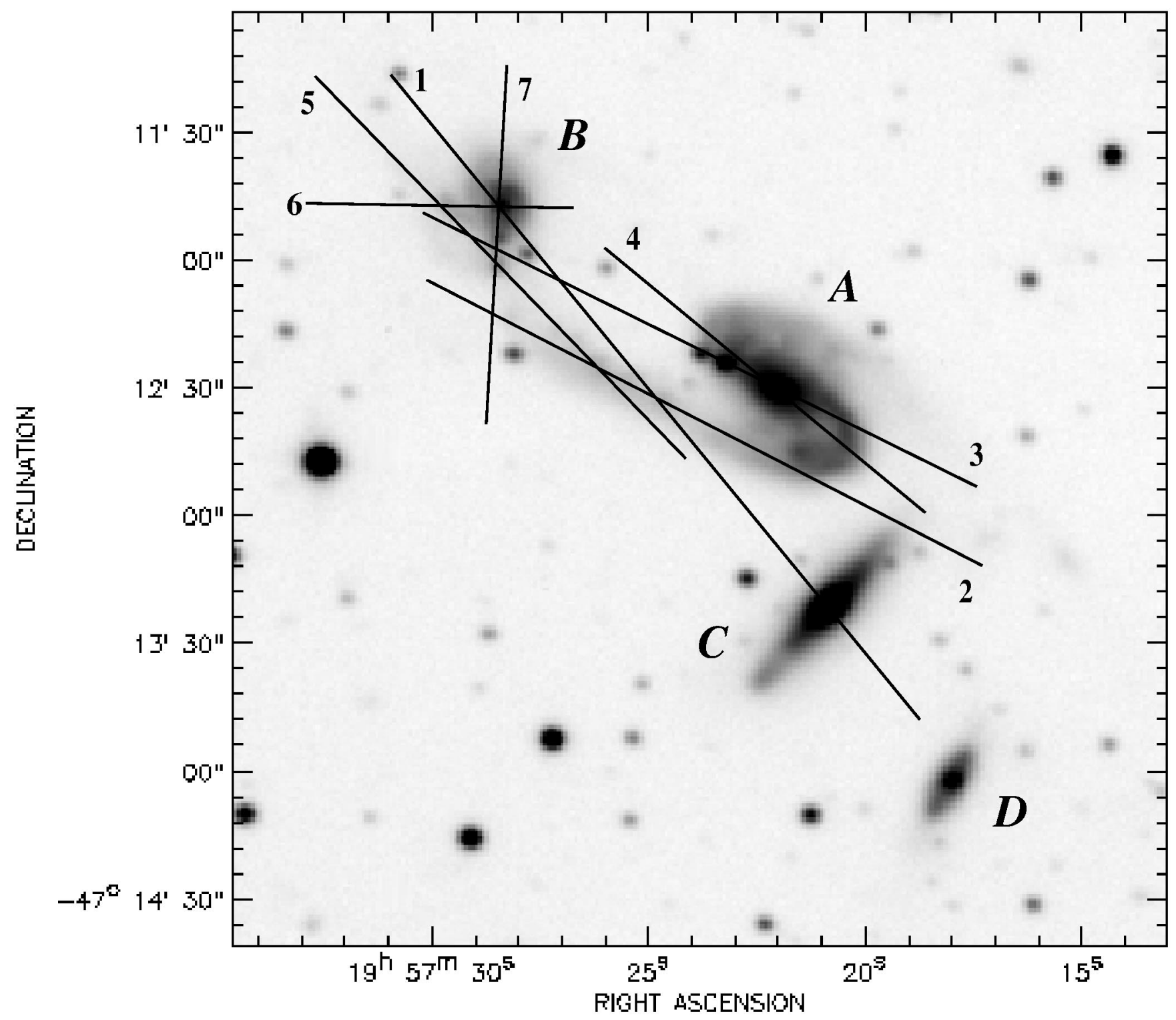

FIG. 5.-Slit positions for the spectra of Table 3. Slit lengths are not to scale

and the southwest sides of the rotation curve for $r \geq 6 \mathrm{kpc}$ (see Fig. 7): to the northeast it shows a strong bump at about $6 \mathrm{kpc}$, whereas the southwest half presents a plateau at about the same distance. A qualitative comparison with numerical simulations (see, e.g., Mihos \& Bothun 1997; Barton, Bromley \& Geller 1999) suggests that the difference is more likely due to tidal rather than intrinsic effects, which perturb more strongly the galactic disk periphery.

The average rotation curve (Fig. 7) was fitted with the Satoh (1980) density law:

$$
V_{c}^{2}(R, Z)=G M R^{2}\left[R^{2}+Z^{2}+a\left(a+2 \sqrt{Z^{2}+b^{2}}\right)\right]^{-3 / 2},
$$

where $V_{c}(R, Z)$ is the circular velocity at position $(R, Z), M$ is the total mass, and $a$ and $b$ are shape parameters with the dimension of length. Following Satoh, a ratio of $b / a \approx 0.1$ corresponds to a disk-dominated distribution, $b / a \approx 1$ to a disk plus a well-developed bulge (Sa-SO, see Satoh 1980) and $b / a \approx 10$ to a near spherical distribution. $\chi^{2}$ fitting was performed by the downhill simplex minimization algorithm ("amoeba") with the IRAF NFIT1D task. The parameters obtained for the fit were $a=6.5 \pm 0.4$ and $b / a=0.3 \pm 0.1$, which indicates a dominant disk with a small to intermediate bulge, in agreement with the RC3 classification. The fit is shown in Figure 7, where the full line represents the best fit and the dashed lines give its lower and upper limits within the observational errors, which in turn furnish $a$ and $b / a$ errors. The derived model total mass is $M_{\text {Satoh }}=(4.4$ $\pm 1.2) \times 10^{11} M_{\odot}$. The Keplerian mass up to the last point on the rotation curve is $3.2 \times 10^{11} M_{\odot}$, broadly in agreement with the more sophisticated fit based on Satoh's model.

NGC 6845 A's mass is comparable to that of Andromeda. Andromeda's brightness is $V=3.44 \mathrm{mag}$ at a distance of 0.7 $\mathrm{Mpc}$, corresponding to $13.5 \mathrm{mag}$ at the distance of NGC $6845 \mathrm{~A}$, and indicating that both galaxies are comparable in their mass-to-luminosity ratio. Nevertheless, the size of NGC $6845 \mathrm{~A}$ is only one-half that of Andromeda. This figure would be difficult to change since, in order to make both galaxies comparable in size, a Hubble constant smaller than $35 \mathrm{~km} \mathrm{~s}^{-1} \mathrm{Mpc}^{-1}$ would be required. It is also difficult 


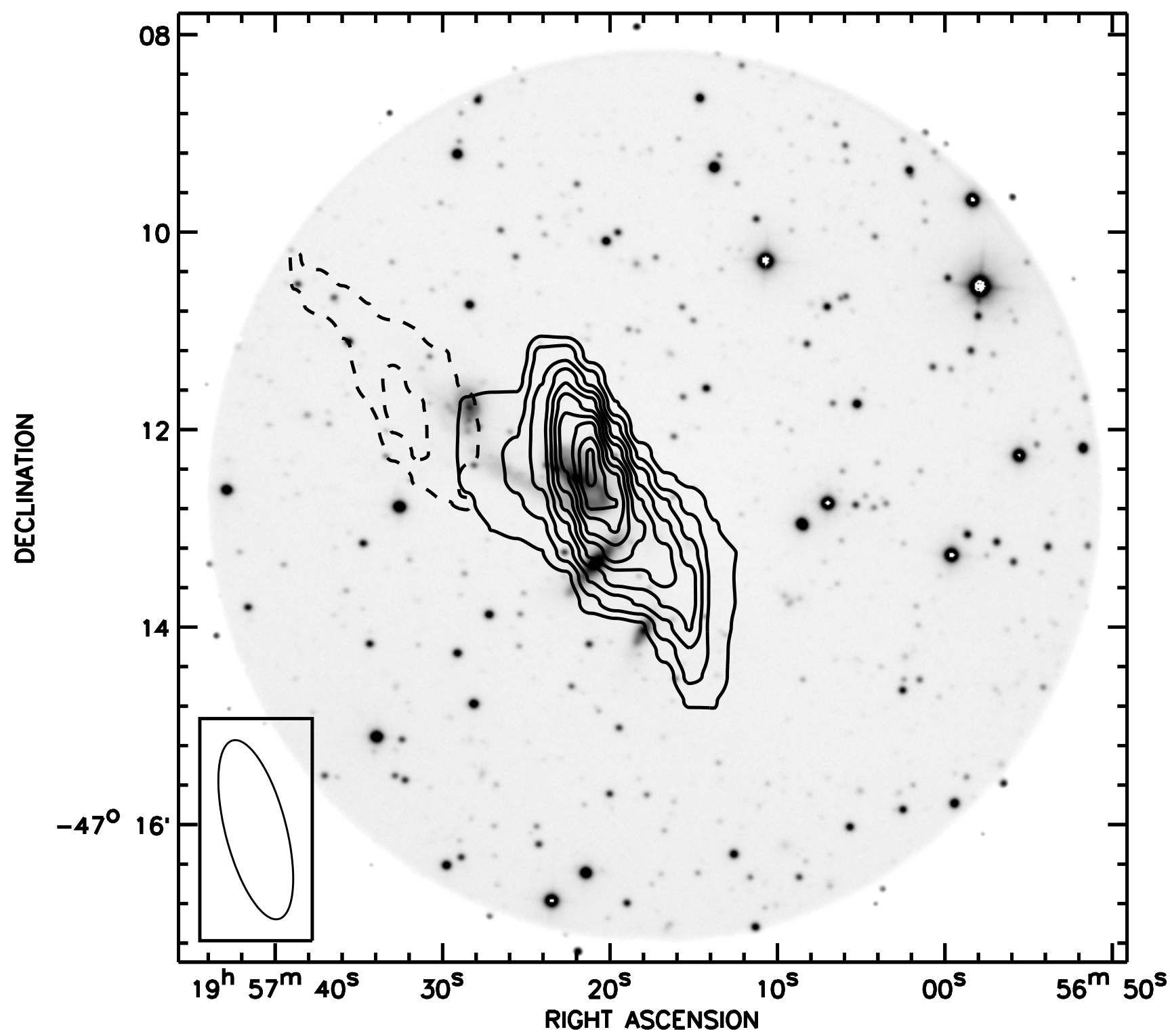

FIG. 6. - H I contour map superimposed on the R image of NGC 6845. The solid lines show H I associated with NGC 6845 A, with count peak flux $=4.08 \times 10^{3} \mathrm{Jy}_{\text {beam }}^{-1} \mathrm{~m} \mathrm{~s}^{-1}$. Ten equally spaced contour levels from $0.4 \times 10^{3}$ to $4.0 \times 10^{3} \mathrm{Jy} \mathrm{beam}^{-1} \mathrm{~m} \mathrm{~s}^{-1}$ are plotted. The dashed lines show $\mathrm{H} \mathrm{I}$ associated with NGC $6845 \mathrm{~B}$ (mean velocity $6700 \mathrm{~km} \mathrm{~s}^{-1}$ ), with count peak flux $=0.95 \times 10^{3} \mathrm{Jy} \mathrm{beam}^{-1} \mathrm{~m} \mathrm{~s}^{-1}$. Contour levels are $0.4 \times 10^{3}$ and $0.8 \times 10^{3}$ $\mathrm{Jy}_{\text {beam }}^{-1} \mathrm{~m} \mathrm{~s}^{-1}$. Beam size is shown at the lower left corner.

to invoke an error in the derived mass for NGC 6845 A due to an error in the disk inclination since, even by assuming an edge-on position for this galaxy, we could not reduce the derived mass by more than a factor of 1.5 . Graham \& Rubin (1973) have already pointed out that NGC 6845 A should have a large mass because of its uncommonly large maximum velocity. We note that their rotation curve extends only up to $\sim 6 \mathrm{kpc}$.

\subsubsection{Tidal Bridge Kinematics}

It is interesting to note that the tidal bridge of NGC 6845 A shows a decreasing trend in radial velocity (see Fig. 3 and Tab 6) starting from its base, where it is connected to the NGC $6845 \mathrm{~A}$ disk near region 8 (at $6660 \mathrm{~km} \mathrm{~s}^{-1}$ ), and passing through $\mathrm{H}$ iI regions 3 , 2, and 1, located more or less in the middle of the tidal bridge (at $6455 \mathrm{~km} \mathrm{~s}^{-1}, 6361 \mathrm{~km}$ $\mathrm{s}^{-1}$, and $6272 \mathrm{~km} \mathrm{~s}^{-1}$, respectively). Regions 4 and 5, which are slightly shifted to the north with respect to the pro- longation of the bridge, surprisingly present radial velocities that agree with this trend $\left(6314 \mathrm{~km} \mathrm{~s}^{-1}\right.$ and $6201 \mathrm{~km} \mathrm{~s}^{-1}$, respectively), strongly suggesting that they are linked to the tidal bridge and not to NGC $6845 \mathrm{~B}$, despite the fact that they are seen projected on top of this object. We also detected $\mathrm{H}$ I on the tidal bridge, with a mean velocity of $6350 \mathrm{~km} \mathrm{~s}^{-1}$, in agreement with the optical results (see next section).

Supposing that the tidal bridge tip follows a parabolic orbit in the plane of NGC 6845 A's disk and its present true velocity is the observed radial velocity of the $\mathrm{H}$ II regions 4 and 5, we obtain a collision age of $\sim 150-300$ Myr. Since $\mathrm{H}$ II regions cannot be older than $10 \mathrm{Myr}$ (see $\S 3.2$ ), this implies that star formation activity occurs inside the tidal bridge, started well after the beginning of the bridge formation.

A possible scenario for the bridge late star formation emerges from the simulations by Barnes \& Hernquist 


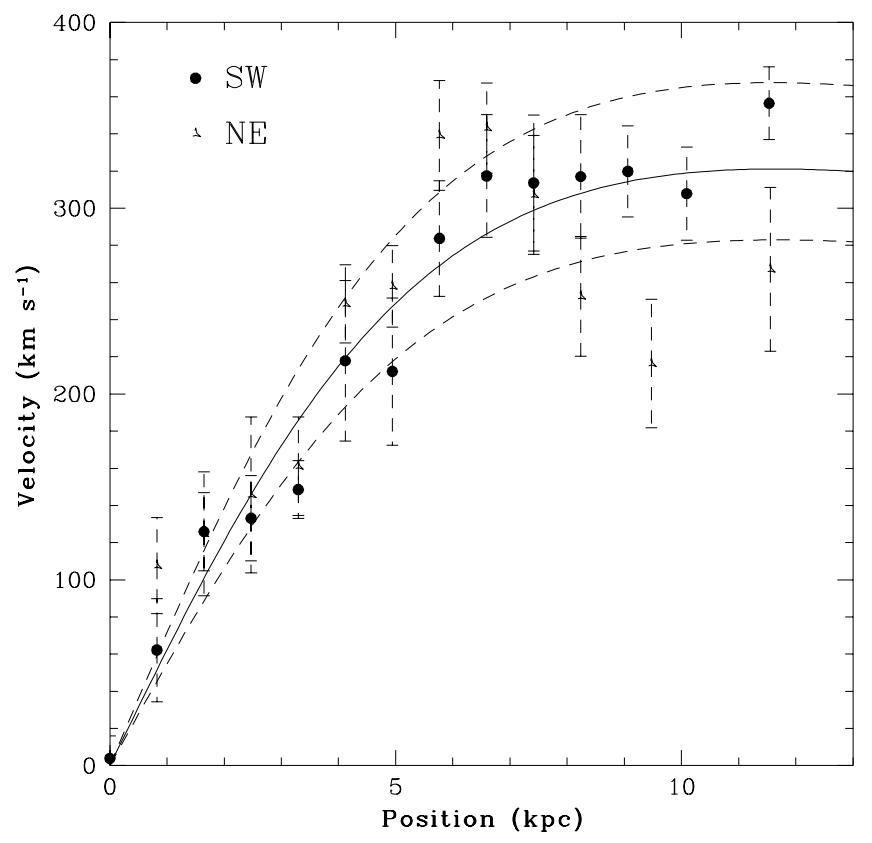

Fig. 7.- Rotation curve of NGC 6845 A. Different symbols are used to distinguish between southwest (filled circles) and northeast (open triangles) directions. The full line gives our best Satoh model fit to the observed data set $\left(\chi^{2}=48.5\right.$ and $\mathrm{rms}=18.1 ; a=6.5 \pm 0.4$ and $\left.b / a=0.3 \pm 0.1\right)$. Dashed lines give the upper and lower limits of the fitted curve within the observational errors.

(1992). These authors found that self-gravitating star clumps form along tidal tails after a collision. The gas clouds torn apart from the galactic disk simultaneously with the stars may fall into these clumps, reaching the density necessary to form stars. Cloud-cloud collisions inside the tidal bridge may be an alternative mechanism to explain the star formation inside the bridge.

\subsubsection{H I Velocity Field of NGC $6845 \mathrm{~A}$}

The isovelocity map of the $\mathrm{H}$ I component associated with NGC 6845 A is shown in Figure 8, superimposed on the $R$-band image.

In Figure 9, we show the $\mathrm{H}$ I position-velocity diagram, obtained by cutting the data cube along the major axis of NGC 6845 A (line of the optical spectrum slit number 4 in Fig. 4). In this figure the center of NGC $6845 \mathrm{~A}$ is at $\delta=-47^{\circ} 12^{\prime} 30^{\prime \prime}$ and $6399 \mathrm{~km} \mathrm{~s}^{-1}$. To the South of the galaxy center, the diagram shows a behavior similar to that of the optical rotation curve (Fig. 7), so we conclude that in this part the $\mathrm{H}$ I kinematics is mainly rotational. To the North, the lower part of this diagram shows a plateau at $6350 \mathrm{~km} \mathrm{~s}^{-1}$, coincident with the tidal bridge velocity. Supposing that the $\mathrm{H} \mathrm{I}$ is in dynamical equilibrium, we derive from its kinematics (Fig. 8) a mass of $1.4 \times 10^{12} M_{\odot}$, about three times higher than the mass derived from the optical data. We attribute this difference to the fact that the $\mathrm{H}$ I can be followed out to larger distances, especially to the SW (the upper part of the position-velocity diagram of Fig. 9), and hence will trace the dark matter associated with the halo of NGC 6845 A. Nevertheless, we have to keep in mind that the $\mathrm{H}$ I kinematics may suffer nonrotational effects. The H I map low spatial resolution does not allow to see the classical butterfly diagram, characteristic of two-dimensional rotational maps. As can be seen in Figure 8, the isovelocity lines are roughly oriented along the beam major axis.

\subsubsection{The Group Kinematical Mass}

Following Binney \& Tremaine (1987, eq. [10-21]), the radial velocity difference among the system members, their absolute luminosities (Table 4), and their projected positions, allow us to derive the group mass-to-luminosity ratio. For the group member velocities we adopt the mean value of the available data (Rose \& Graham, RC3, and ours, see Table 4).

An important source of error in determining $M / L$ is the high inclination of the $\mathrm{C}$ component and, to a lesser extent, that of the A component, the group's most massive galaxies. The inclination of each group member was obtained as described in $\S 3.4 .1$ for NGC 6845 A. The Holmberg (1958) correction was applied to the measured isophotal axis ratio $b / a$ by the formula $\cos ^{2}(i)=\left[(b / a)^{2}-q_{0}^{2}\right] /\left(1-q_{0}^{2}\right)$ in order to obtain the galaxies' inclinations (see Table 4), where $q_{0}=0.2$ (Kodaira \& Watanabe 1988). This correction is especially important for NGC $6845 \mathrm{C}$. We obtained for this galaxy $b / a=0.22 \pm 0.05$, measured at the isophotal level 25 mag $\operatorname{arcsec}^{-2}$. Galactic extinction was corrected with the standard absorption law (Burstein \& Heiles 1984). In order to obtain the galaxies' face-on magnitudes we corrected internal absorptions by the empirical (RC3) formula $A(i)=A(0)+\alpha \log \sec (i)$, which was evaluated following two different prescriptions of the values of $A(0)$ and $\alpha$. The first one is from Rubin et al. (1982), where $A(0)=0$ and $\alpha=1.9$, leading to $(M / L)_{1}=43.5 \pm 2.0$ (solar units), with the uncertainties estimated from the errors in $\alpha( \pm 0.16$; Kodaira \& Watanabe 1988) and $i$ (Table 4). The second prescription is that of $\mathrm{RC} 3$, where $A(0)=0.12$ and $\alpha=0.8$ for NGC 6845 A and $A(0)=0.1$ and $\alpha=0.7$ for galaxies B, $\mathrm{C}$ and $\mathrm{D}$. In this case we obtained $(M / L)_{2}=66.4 \pm 2.0$. Rose \& Graham (1979) estimated $M / L$ in the range $28-49$, also depending on the source for Galactic and internal extinction corrections. Our $(M / L)_{1}$ is well inside their range of values, while $(M / L)_{2}$ is higher than their highest $M / L$.

Although large, the difference between $(M / L)_{1}$ and $(M / L)_{2}$ points to very similar total masses of the system $\left(1.0 \pm 0.2 \times 10^{13} \quad M_{\odot}\right.$ and $8.7 \pm 0.2 \times 10^{12} M_{\odot}$ respectively). The luminous mass of the system cannot account for more than $\approx 1.6 \times 10^{12} M_{\odot}$ (scaling the mass of the galaxies by their luminosities with respect to the mass and luminosity of NGC $6845 \mathrm{~A}$ ), indicating that the group's dark matter mass is approximately 5 times higher than its luminous mass. The whole system contains almost 9 times more dark matter than was thought to be associated with the halo of NGC $6845 \mathrm{~A}$, as derived in the previous section from $\mathrm{H}$ I kinematics.

\section{SCENARIOS FOR THE COLLISION}

Strong, well-developed tidal bridges and tails are characteristic of galaxy encounters in which the disk of the perturbed galaxy and the orbit of the perturber rotate in the same sense, i.e., a prograde orbit, and the angle between the main galaxy disk and the orbital plane is small (see, e.g., Toomre \& Toomre 1972; Negroponte \& White 1983; Howard et al. 1993). In prograde encounters, tidal arms curve in a sense opposite to the disk rotation: they are trailing. Consequently, we can infer that NGC 6845 A disk rotates counterclockwise ( $\$ 3.4 .1)$ and its southeast side is preceding, indicating that the tidal bridge side is the near one.

Graham \& Rubin (1973) already noted as curious the fact that, because of the relative position and radial velocities of 


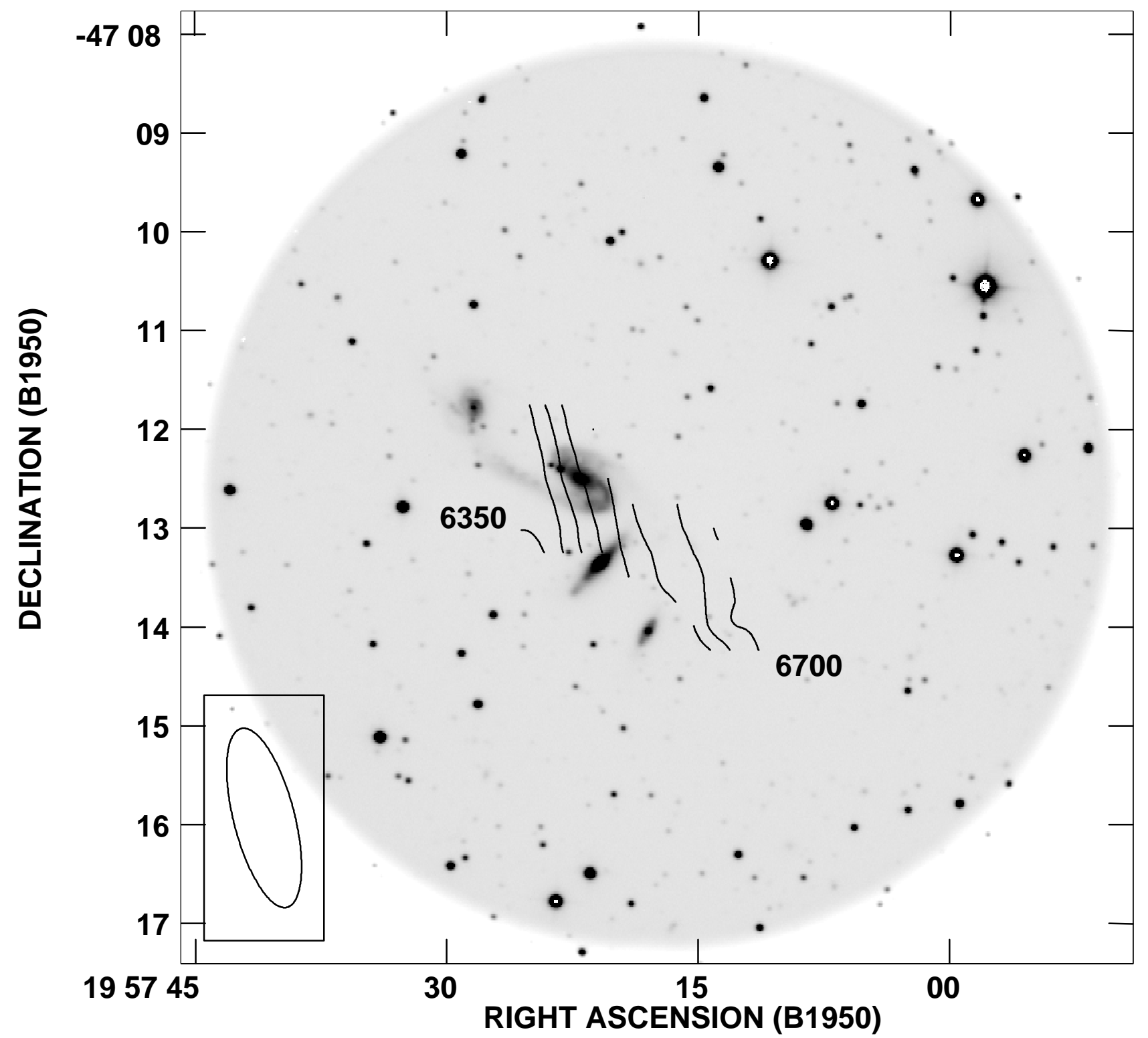

FIG. 8.-H I velocity map superimposed on the R image of NGC 6845 . Velocity levels are from 6350 to $6700 \mathrm{~km} \mathrm{~s}^{-1}$ in intervals of $50 \mathrm{~km} \mathrm{~s}^{-1}$

galaxies $\mathrm{A}$ and $\mathrm{B}$, any simple geometry would indicate that the $\mathrm{B}$ orbit is retrograde. However, as mentioned by these authors, the case might be similar to that of M 51/NGC 5195, for which Toomre \& Toomre (1972) found it possible to obtain a direct sense of rotation if the orbital eccentricity of NGC 5195 is large and if the orbit is highly inclined to the plane of M 51. From this point of view, galaxy $\mathrm{C}$ would be a good candidate for the strong interaction. Nevertheless, this galaxy presents itself as a symmetric disk up to faint brightness levels. Modern numerical simulations of similar disk encounters (see, e.g., Barnes 1992) show that a collision between two large disks, like those of NGC $6845 \mathrm{~A}$ and $\mathrm{C}$, close enough to produce the NGC $6845 \mathrm{~A}$ tidal bridge, would necessarily strongly distort NGC 6845 C disk as well.

In order to understand the dynamical evolution of this group, two of us (Rodrigues \& Dottori 1999) are developing extensive numerical simulations with the TreeSPH code (Hernquist \& Katz 1989) on a CRAY T94 computer (CESUP-UFRGS). From this work we advance some pre- liminary results. In Figures 10 and 11, we show simulations of collisions of A with C and A with B. Galaxy models were constructed following the general prescription of Kuijken \& Dubinski (1995). The galaxy A model is such that its rotation curve presents a circular velocity of $300 \mathrm{~km} \mathrm{~s}^{-1}$ at 6 $\mathrm{kpc}$ and is approximately flat up to the cutoff radius $(20$ $\mathrm{kpc}) .10 \%$ of the disk mass is composed of gas particles, whose density profile is more extended than that of the stellar disk. For galaxy $\mathrm{C}$ we adopted the same model as of $\mathrm{A}$, but without gas, since this galaxy does not present $\mathrm{H}$ I or emission lines in its optical spectrum. Galaxy models A and $\mathrm{C}$ each consist of 6000 particles with a mass of $3.5 \times 10^{11}$ $M_{\odot}$ per galaxy. Galaxy B is represented by a point mass of $1 / 4$ of model A's mass.

Figure 10 shows the evolution of the encounter of models $\mathrm{A}$ and $\mathrm{C}$, which approach along a parabolic orbit with a perigalactic distance $q=12 \mathrm{kpc}$. The first row shows the time evolution of the stellar component, while the second row shows the same for the gas component. For ease of comparison between simulation and observations we used a 


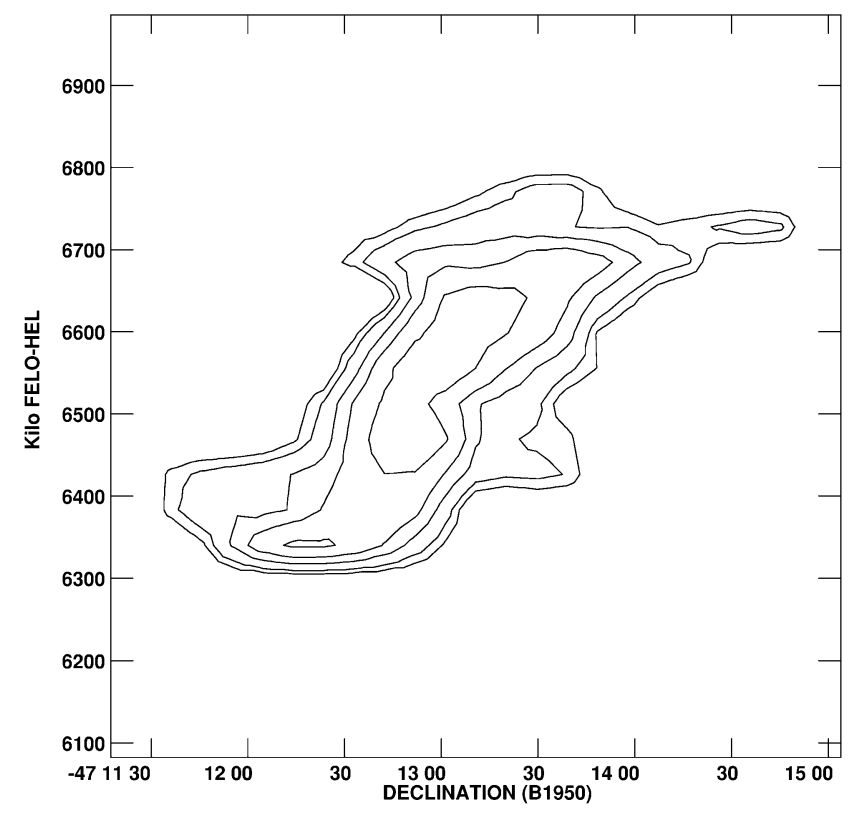

Fig. 9.-NGC 6845 A H I position-velocity diagram, obtained by cutting the data cube along the galaxy major axis, coincident with the slit number 4 in Fig. 4, the one used to obtain the optical rotation curve of NGC 6845 A (Fig. 7). The $x$-axis corresponds to the cut projection on the declination direction. Contour levels are drawn at 0.30, 0.45, 0.75, 1.0, and $1.5 \mathrm{~K}$.

coordinate system in which the $z$-axis corresponds to the line of sight (with positive values toward the observer), the $x$-axis is the east-west direction (positive to the west), and the $y$-axis is the north-south direction (positive to the north). In this system, the orbit of $\mathrm{C}$ around $\mathrm{A}$ has its pericenter in the direction of the vector $(-3,-10,10)$ and the orbital plane is defined by the orbital spin vector, parallel to $(10,3,6)$. We identify the third frame of Figure $11(104 \mathrm{Myr}$ after the perigalacticum) as the most similar to the system at its present stage. A striking result in favor of this scenario is the presence of a stellar condensation similar to that observed to the right of galaxy C's northwest extreme. Model C's southeast extreme bends to the east, which is also observed to the faintest brightness levels in galaxy $\mathrm{C}$ (Fig. 1). The simulation also clearly shows that this collision produces a strong tidal feature similar to the NGC $6845 \mathrm{~A}$ tidal bridge with a condensation near its extreme that resembles $\mathrm{H}$ II regions 4 and 5, but nothing as large as galaxy B is produced out of this encounter, so the position of galaxy B at the tidal bridge extreme would be a projection within this scenario. Against this scenario the simulation shows that about $10 \%$ of the gas originally belonging to galaxy A would be captured by galaxy $\mathrm{C}$, which is observed neither as $\mathrm{H}$ II nor as $\mathrm{H}$ I. The simulation also shows that stars stripped from A should form a stellar feature perpendicular to C's disk, also not detected in the optical images, which presents a high degree of symmetry to intermediate brightness levels.

Figure 11 shows the encounter of model A with the point mass $B$, under a prograde hyperbolic orbit with eccentricity $e=2$ and perigalactic distance $q=8 \mathrm{kpc}$. The pericenter is in the direction $(20,-8,-10)$ and its orbital spin vector is parallel to $(8,20,0)$. The third frame $(145 \mathrm{Myr}$ after the perigalacticum, shown in the second frame) shows the best match of the model to the system at present stage. In this frame galaxy $B$ is receding with respect to $A$ at $300 \mathrm{~km} \mathrm{~s}^{-1}$. The more striking feature of this simulation is the morphology of the tidal bridge, which is straight and narrow and shows condensations of gas and stars at its base and middle part, resembling the observed tidal bridge morphology. Also the tidal tail is diffuse, as in the real situation. Against this scenario the simulation shows that the tidal tail points in a direction different from the observed one and does not bifurcate.

In spite of both scenarios' pros and cons, the collision of A and B seems to be more appropriate to explain the tidal bridge structure. Although some features associated with galaxy $\mathrm{C}$ can be regarded as tidally produced, it cannot be

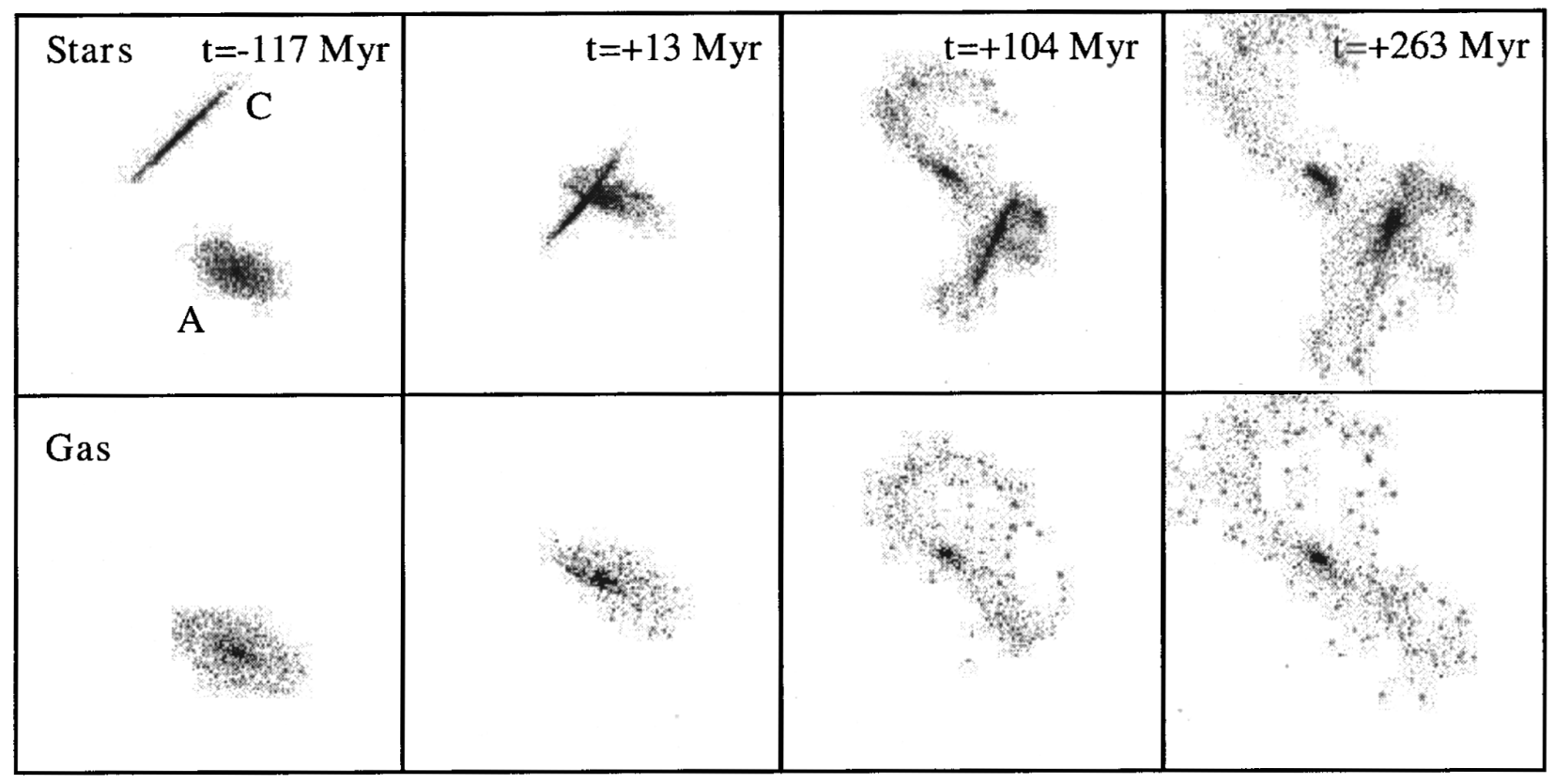

FIG. 10.-Evolution of the encounter of model galaxies A (with gas) and C (without gas), viewed in the sky plane. The top row shows only the stellar content, while the bottom row shows only gas particles. The frames measure $112 \mathrm{kpc}$ on a side, and time is shown in the upper right. 


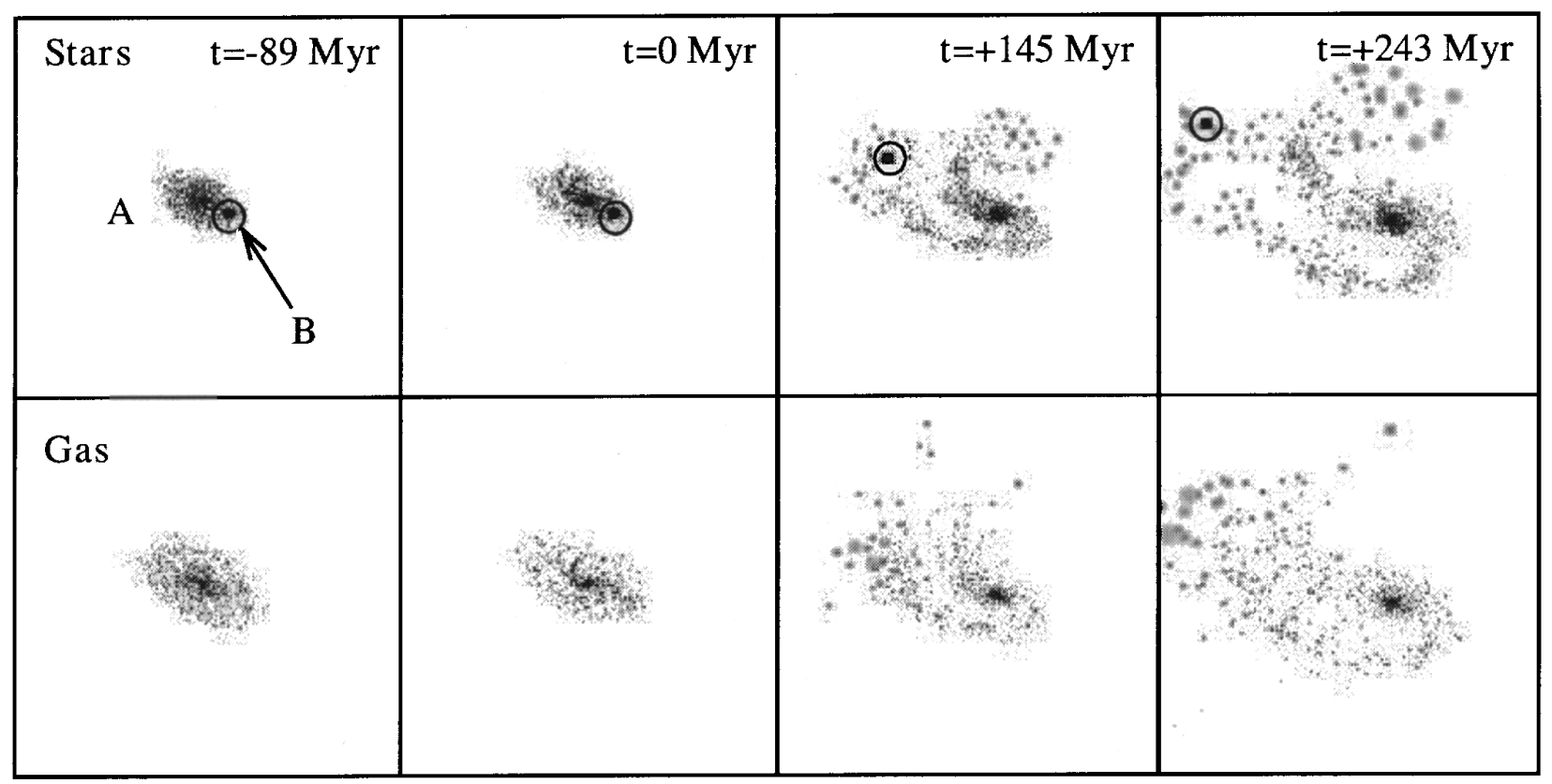

FIG. 11.-Evolution of the encounter of galaxy model A and a point mass representing galaxy B, viewed in the sky plane. The top row shows the stellar content, while the bottom row shows the gas particles. The frames measure $112 \mathrm{kpc}$ on a side, and time is shown in the upper right.

ignored that $\mathrm{C}$ lacks other important features that it should show if it were the main perturber. Perhaps a three-body interaction with B producing the tidal bridge would be more suitable to explain the richness of features observed in this group.

\section{CONCLUDING REMARKS}

The system NGC 6845 is a compact group in which NGC $6845 \mathrm{~A}$ presents a strong tidal bridge, signature of a close collision.

We present the first atomic hydrogen observation of NGC 6845, obtaining an $\mathrm{H}$ I content 5 times higher than in the case of the Milky Way. The H I distribution can be separated into two components, one related to NGC 6845 A, sporting well-developed tidal features, and the second one with velocity similar to that of NGC $6845 \mathrm{~B}$. Components $\mathrm{C}$ and $\mathrm{D}$ do not present $\mathrm{H}$ I up to our detection limit. This fact argues against galaxy $\mathrm{C}$ being the main perturber of $\mathrm{A}$, since, as shown by numerical simulations, gas originally belonging to A should be transferred to $\mathrm{C}$.

NGC 6845 A's optical rotation curve is asymmetric, with an uncommonly high maximum circular velocity, especially to the southwest side. Satoh model fitting to the rotation curve indicates the presence of a dominant disk and a small to intermediate bulge, in agreement with the morphological classification of this galaxy. The derived mass is $M_{\text {Satoh }}=$ $(4.4 \pm 1.2) \times 10^{11} M_{\odot}$. The $B-I$ color map indicate the presence of dust in front of the southwest side of the bar, pointing to a trailing bar.

The $B-I$ color map also shows that the bridge is bluer than NGC 6845 A's inner disk. H II regions brighter than those of the Antennae and NGC 7252 tidal tails have formed at the tip and at the base of the tidal bridge. Three $\mathrm{H}$ II regions midway along the tidal bridge are brighter than NGC 3603 (the brightest known H II region in our Galaxy). The kinematics of $\mathrm{H}$ II regions 4 and 5 indicate that they are more likely connected to the tidal bridge than to NGC 6845 $\mathrm{B}$, as their projected position may suggest. They present luminosities similar to that of 30 Doradus. A simple model for the kinematics of the tip of the bridge as well as numerical simulations indicates that star formation is occurring along this structure $10^{8} \mathrm{yr}$ or more after the perturber's closest approach.

The group kinematics calls for dark matter within the system at about 5 times its luminous mass. The group amount of dark matter would be 9 times higher than the halo mass of the NGC $6845 \mathrm{~A}$, provided that the $\mathrm{H} \mathrm{I}$ is in dynamical equilibrium inside the galaxy; this in turn would be 3 times higher than the galaxy kinematical mass derived from the optical rotation curve.

Since abundant signs of interactions are present in galaxy $\mathrm{B}$, and to a lesser extent in C, we appealed to $N$-body simulations in order to disentangle which of the galaxies, B or C, would be the main perturber of A. Both scenarios for the tidal disruption of galaxy A present pros and cons, but the bridge, which is the most conspicuous tidal feature of this galaxy, seems to be better reproduced by the simulation of the encounter of galaxies A and B. The fact that neither $\mathrm{H} \mathrm{I}$ nor $\mathrm{H}$ II-which should have been stripped from galaxy $\mathrm{A}$-are detected in galaxy $\mathrm{C}$ reinforces this scenario. Perhaps a three-body interaction would be a more appropriate scenario to explain the group dynamical evolution, but it is difficult to model because of the large number of degrees of freedom involved in such a collision.

We are grateful to Lars Hernquist, who kindly made available the TreeSPH code, to Ruben Diaz, who carried out the observations at Bosque Alegre, and to Leo Girardi, who kindly provided his evolutionary synthesis models. The authors gratefully acknowledge the NRAO for allocating observing time and for support. We also thank the staffs of CASLEO and Bosque Alegro observatories. Numerical calculations were done at the CESUP-UFRGS national supercomputing center. This work was supported in part by the Brazilian institutions CNPq and FINEP. 


\section{REFERENCES}

Arp, H. C., \& Madore, B. F. 1987, A Catalogue of Southern Peculiar Galaxies and Associations (Cambridge: Cambridge Univ. Press)

Barnes J. 1988, ApJ, 336, 655 . 1992, ApJ, 393, 484

Barnes, J., \& Hernquist, L. 1992, Nature, 360, 715

Barton, E. J., Bromley, B. C., \& Geller, M. J. 1999, ApJ, 511, L25

Bertelli, G., Bressan, A., Chiosi, C., Fagotto, F., \& Nasi, E. 1994, A\&AS, 106,275

Binney, J., \& Tremaine, S. 1987, Galactic Dynamics, Princeton Series in Astrophysics (Princeton: Princeton Univ. Press)

Burstein, D., \& Heiles, C. 1984, ApJS, 54, 33

de Vaucouleurs, G., de Vaucouleurs, A., Corwin, H. G., Jr., Buta, R. J.,

Paturel, G., \& Fouqué, P. 1991, Third Reference Catalogue of Bright Galaxies (New York: Springer)(RC3)

Duc, P.-A., Brinks, E., Wink, J., \& Mirabel, I. F. 1997, A\&A, 326, 537

Duc, P.-A., \& Mirabel, I. F. 1994, A\&A, 289, 83

Elmegreen, B. G., Kaufmann, M., \& Thomasson, M. 1993, ApJ, 412, 90

Eneev, T. M., Kozlov, N. N., \& Sunyaev, R. A. 1973, A\&A, 22,41

Graham, J. A., \& Rubin, V. C. 1973, ApJ, 183, 19

Hernquist, L., \& Katz, N. 1989, ApJS, 70, 419

Hibbard, J. E., Guhathakurta, P., van Gorkon, J. H., \& Schweizer, E. 1994, AJ, 107, 67

Holmberg, J. H. 1958, Med. Lunds Astron. Obs. Ser. II, 136

Howard, S., Keel, W. C., Byrd, G., \& Burkey, J. 1993, ApJ, 417, 502

Kennicutt, R. C., \& Chu, Y.-H. 1988, AJ, 95, 720

Kennicutt, R. C., Edgar, B. K., \& Hodge, P. W. 1989, ApJ, 337, 761

Klemola, A. R. 1969, AJ, 74, 804

Kodaira, K., \& Watanabe, M. 1988, ApJ, 96, 1593
Kuijken, K., \& Dubinski, J. 1995, MNRAS, 277, 1341

Landolt, A. U. 1992, AJ, 104, 372

Melnick, J., Tapia, M., \& Terlevich, R. 1989, A\&A, 213, 89

Mihos, J. C., \& Bothun, G. D. 1997, ApJ, 481, 471

Mirabel, I. F., Dottori, H., \& Lutz, D. 1992, A\&A, 256, L19

Mirabel, I. F., Lutz, D., \& Maza, J. 1991, A\&A, 243, 367

Negroponte, J., \& White, S. D. M. 1983, MNRAS, 205, 1009

Osmer, P. S., Smith, M. G., \& Weedman, D. W. 1974, ApJ, 192, 279

Pastoriza, M. G., Dottori, H. A., Terlevich, E., Terlevich, R., \& Diaz, A. 1993, MNRAS, 260, 177

Rodrigues, I., \& Dottori, H. 1999, in preparation

Rose, J. A. 1979, ApJ, 231, 10

Rose, J. A., \& Graham, J. A. 1979, ApJ, 231, 320

Rubin, V. C., Ford, W. K., Jr., Thonnard, N., \& Burstein, D. 1982, ApJ, 261, 439

Rush, B., Malkan, M. A., \& Spinoglio, L. 1993, ApJS, 89, 1

Salo, H. 1991, A\&A, 243, 118

Sanders, D. B., \& Mirabel, I. F. 1996, ARA\&A, 34, 749

Satoh, C. 1980, PASJ, 32, 41

Schweizer, F. 1978, in IAU Symp. 77, Structure and Properties of Nearby Galaxies, ed. E. M. Berkhuijsen \& R. Wielebinski (Dordrecht: Reidel), 279

Stockton, A. 1974, ApJ, 187, 219

Stone, R. P. S., \& Baldwin, J. A. 1983, MNRAS, 204, 347

Toomre, A., \& Toomre, J. 1972, ApJ, 178, 623

Wright, A. E. 1972, MNRAS, 157, 309

Zwicky, F. 1956, Ergeb. Exakten Naturwiss., 29, 344 